\title{
Das Competências Quantitativas Iniciais para o Conceito de Número Natural: Quais as Trilhas Possíveis?
}

\author{
From Quantitative Abilities to Natural Number Concept: \\ What are the Possible Pathways?
}

\author{
Heloiza H. Barbosa* \\ Universidade Federal de Santa Catarina, Florianópolis, Brasil
}

\begin{abstract}
Resumo
A presente revisão tem o objetivo de analisar as propostas apresentadas por estudos na área da cognição matemática sobre as habilidades quantitativas iniciais dos bebês e como estas habilidades iniciais podem levar à construção do conceito de número natural. A partir das evidências, o presente artigo discute se a ideia de número natural é inata, ideia defendida pela posição nativista original, ou se é derivada de um processo de desenvolvimento. Assim como também, apresenta dados de estudos recentes que sugerem ser o conhecimento de número natural um processo de desenvolvimento cognitivo gradual e de domínio genérico. Embora, mais estudos sejam necessários para se firmar esta última conjectura.
\end{abstract}

Palavras-chave: Cognição matemática, número, formação de conceito.

\begin{abstract}
The present review aims to analyze the proposal put forward by researches on mathematical cognition about the initial quantitative abilities in human infants. Also, it analyses how the human infants may construct the concept of natural number upon these initial abilities. From the data presented, the paper discusses whether the idea of number has an innate basis, which is an original nativist's position, or a developmental process. Additionally, it presents data from new studies that point towards a cognitive process in the natural number concept that goes from general to specific domains. However, more studies are needed to support this proposal.

Keywords: Mathematical cognition, number, concept formation.
\end{abstract}

Jean Piaget e a pesquisadora Alina Szeminska (Piaget, $1952)^{1}$, foram os primeiros a estudar, por meio de metodologias experimentais, a complexidade envolvida na formação do conceito de número em crianças de idade pré-escolar (de quatro a seis aos de idade). $\mathrm{O}$ foco de pesquisa de Piaget e Szeminska estava em procurar sinais de compreensão da quantificação numérica em crianças da referida faixa etária (a partir de 4 anos), descrevendo e interpretando, então, diversos esquemas e relações prénuméricas que estariam na raiz da construção da noção de número natural. Os estudos destes pesquisadores de Genebra deram força à hipótese que a noção de número é derivada de um longo processo de construção que emerge da interação entre o sujeito, o mundo físico e as realidades temporais.

\footnotetext{
* Endereço parara correspondência: Departamento de Estudos Especializados, Centro de Ciências da Educação, Universidade Federal de Santa Catarina, Campus Universitário Trindade, Florianópolis, SC, Brasil 88010 970. E-mail: heloiza@ced.ufsc.br

${ }^{1}$ Alina Szeminska não teve o seu nome incluído na tradução do livro sobre o desenvolvimento dos conceitos numéricos na versão para o idioma inglês.
}

Essa hipótese foi amplamente contestada por pesquisadores norte-americanos e anglo-saxônicos por diferentes razões. Essa discordância forjou a criação de uma agenda de pesquisa com o objetivo de coletar dados para: (a) questionar o caráter construcionista do conceito de número; (b) comprovar a existência do conceito de número natural em idades mais precoces e, assim, desafiar os marcos cronológicos propostos por Piaget e Szeminska, e (c) mostrar um papel mais relevante da contagem oral na conceituação de número ${ }^{2}$. Assim, podemos dizer que os estudos pós-piagetianos, principalmente os conduzidos nos Estados Unidos e no Reino Unido, são marcados pelo debate em torno de duas questões: (a) a natureza do conceito de número; ou seja, se este conceito é inato ou se é construído e (b) o papel da contagem na construção do conceito de número.

A publicação do livro The Child's Understanding of Number (Gelman \& Gallistel, 1978), é um marco nas 
pesquisas na área da cognição matemática que, então, começam a delinear uma criança, a qual, desde a mais tenra infância, possui habilidades quantitativo-numéricas guiadas por estruturas conceituais de número de natureza inata. Portanto, muito diferente da criança em desenvolvimento descrita por Piaget e Szeminska (Piaget, 1952). A pletora de estudos produzidos desde então, sugere que a criança desde muito cedo, até mesmo antes da aquisição da linguagem verbal, é capaz de discriminar quantidades, comparar conjuntos e antecipar resultados de transformações quantitativas. A criação de novas e engenhosas metodologias para estudo com crianças pré-verbais (bebês) possibilitou a investigação destas habilidades.

Embora pareça haver consenso entre pesquisadores de que as crianças desde muito cedo são capazes de produzir representações de informações quantitativas, não há ainda forte concordância no que diz respeito à natureza destas representações: são estas representações baseadas em conhecimento de natureza conceitual inata? $\mathrm{Ou}$, são estas representações frutos de um complexo processo de desenvolvimento?

Há pesquisadores que argumentam em favor da natureza inata do conhecimento matemático. Entre esses pesquisadores há controvérsias sobre os mecanismos cognitivos que sustentam tal conhecimento. Alguns argumentam que as crianças formam representações mentais quantitativas baseadas em conhecimento inato de número (Butterworth, 1999; Carey, 1991; Carey \& Spelke, 1994; Dehaene, 1997; Gelman, 1991; Gelman \& Gallistel, 1978). Outros propõem que estas representações são baseadas em mecanismos cognitivos inatos de detecção de magnitudes e individuação de objetos (Carey, 2008; Cordes \& Brannon, 2008; Feigenson \& Carey, 2003; Feigenson, Carey, \& Spelke, 2002; Le Corre \& Carey, 2007; Uller, Carey, Huntley-Fenner, \& Klatt, 1999; Van de Walle, Carey, \& Prevor, 2000).

De outro lado, há os pesquisadores que argumentam em favor de um processo multifatorial, complexo e gradual de desenvolvimento da noção de número. Para esses pesquisadores, em concordância com a teoria piagetiana, o foco está na elaboração da ideia de número a partir das competências elementares de quantificação. No processo de elaboração dessa ideia de número, as representações mentais produzidas pelas crianças são baseadas em processos cognitivos gerais (como por exemplo, o processo da visão, da memória, da percepção tátil dos objetos) sem atrelamento específico a conhecimentos de base numérica, pelo menos inicialmente. Assim, para esses pesquisadores, a construção plena de um conceito de número que englobe a complexidade de entender o número natural nas dimensões cardinal, ordinal e nominal deriva de um longo processo de desenvolvimento (Clearfield \& Mix, 1999, 2001; Huttenlocher, Duffy, \& Levine, 2002; Mix, 2002; Mix, Hutenllocher, \& Levine 2002a, 2002b; Mix \& Sandhofer, 2007; Wiese, 2003).
Esta falta de consenso deixa claro que, no campo da investigação sobre o desenvolvimento da cognição matemática em crianças, há vários caminhos para se chegar do ponto $A$ ao ponto $B$. Ou seja, das habilidades iniciais ao conceito de número natural, vistos em crianças maiores e em adultos, talvez haja múltiplas trilhas. Mas, considerando os resultados mostrados por estudos recentes com bebês e animais, devemos perguntar: são as representações mentais produzidas pelo bebê indubitavelmente baseadas em conhecimentos numéricos? Ou ainda, como o bebê constrói conceito de número natural a partir de habilidades iniciais?

Neste artigo, revisaremos as evidências resultantes de estudos com bebês e animais infra-humanos que focam o desenvolvimento do conceito de número natural.

\section{São Numéricas as Representações Quantitativas Não-Verbais?}

Tem sido divulgado em revistas populares, Veja, Isto É, Nova Escola e Ciência Hoje, que os bebês humanos têm habilidades matemáticas surpreendentes como cálculo, identificação de diferentes magnitudes e comparação. Notícias como estas demandam uma análise crítica dos estudos que demonstraram tais habilidades. São os bebês humanos realmente capazes de reconhecer diferentes magnitudes com base em conhecimentos de natureza inata de número? Os bebês sabem que o numeral dois é mais que um, e que dois é menos que três?

Para estudar o desenvolvimento quantitativo-numérico em bebês, pesquisadores inicialmente tiveram que criar novas metodologias que se adequassem à idade da população estudada. Metodologias comumente usadas nestas investigações são a metodologia da habituação e a metodologia da "busca manual do objeto que falta". Os dados das pesquisas com bebês utilizando ambas as metodologias mostraram que os bebês são sensíveis às informações quantitativas presentes no mundo físico. Por exemplo, usando a metodologia da busca manual e manipulando duas condições experimentais: (a) coerência (Ex: dois objetos inseridos e dois objetos retirados) e (b) incoerência (Ex: dois objetos inseridos e um objeto retirado), os pesquisadores Van de Walle et al. (2000) demonstraram que os bebês conseguem detectar, memorizar e perceber alteração numérica ${ }^{3}$. Nesse experimento, os pesquisadores inseriram duas bolas dentro de uma caixa e, em seguida, retiraram somente uma bola de dentro desta mesma caixa (situação de incoerência; a outra bola ficou escondida em um fundo falso). Depois disso, aos bebês foi dada a caixa para exploração manual. Este experimento demonstrou que os bebês passam mais tempo explorando e buscando dentro da caixa nesta situação de incoerência do que na situação de coerência.
3 "Alteração numérica" foi a terminologia usada pelos pesquisadores para descrever sua conclusões. 
De forma mais robusta, os estudos usando metodologias de habituação demonstraram que os bebês são capazes de identificar mudanças numéricas em conjuntos com pequenas quantidades (Antell \& Keating, 1983; Starkey \& Cooper, 1980; Starkey, Spelke, \& Gelman, 1990; Strauss \& Curtis, 1981, 1984) e em conjuntos com grandes quantidades (Xu \& Spelke, 2000). E, ainda, são capazes de parear de forma equivalente o número de sons ouvidos com o número de objetos de um conjunto (Starkey $\&$ Spelke, 1983) e demonstram conhecimento de resultados de adições e subtrações simples (Wynn, 1992b, 1992c).

Embora não haja dúvidas de que os bebês humanos são capazes de apreender informação quantitativa do seu ambiente, há inúmeras questões pendentes quanto à natureza das representações mentais produzidas a partir destas informações e quanto aos mecanismos cognitivos que possibilitam a emergência e operacionalização de tais habilidades em bebês.

A abordagem inatista original ${ }^{4}$ afirma que o desenvolvimento do conceito de número envolve o movimento de acesso a conhecimentos matemáticos inatos localizados em módulo cerebral específico para números (Butterworth, 1999; Carey, 1991; Carey \& Spelke, 1994; Dehaene, 1997; Gelman, 1991; Gelman \& Gallistel, 1978; Gelman \& Meck, 1983; Spelke \& Dehaene, 1999; Spelke \& Tsivkin 2001; Starkey \& Spelke, 1983; Wynn, 1992b, 1992c, 1998a, 1998b). Assim, para esses pesquisadores, os bebês prestam atenção em informações numéricas ao redor deles porque seus cérebros são equipados desde o nascimento para fazê-lo.

Mas um estudo de Clearfield e Mix (1999) inaugurou uma nova agenda de pesquisa e tornou-se um marco importante na área da cognição matemática. Neste estudo, as autoras Clearfield e Mix enfraqueceram o argumento inatista ao mostrar que os dados dos estudos de habituação, até então produzidos, não desambiguavam os fatores perceptivos dos fatores numéricos. Para isso, as autoras habituaram bebês entre seis a oito meses de idade a conjuntos com dois ou três quadrados que possuíam uma área de contorno total invariante. Os bebês, então, foram testados sob duas condições experimentais. $\mathrm{Na}$ primeira, os bebês foram apresentados a conjuntos com o mesmo número de quadrados, mas com uma área de contorno nova, em relação ao estímulo da habituação. $\mathrm{Na}$ segunda condição, os bebês foram apresentados a conjuntos com novos números de quadrados, mas com a mesma área de contorno, também em relação ao estímulo da habituação. Os resultados mostraram que os bebês olharam por mais tempo para as mudanças de tamanho na área de contorno do que para as mudanças numéricas. Assim, os dados de Clearfield e Mix claramente demonstraram que quando os experimentos controlavam as

${ }^{4}$ Uso o termo "original" para enfatizar ao leitor que esta abordagem sofrerá alterações nos estudos mais recentes que serão vistos no decorrer deste artigo. variáveis perceptivas separando-as das variáveis numéricas, os bebês mostravam preferência pelas variáveis de natureza perceptiva e não pelas variáveis de natureza numérica.

Os resultados de Clearfield e Mix (1999) foram corroborados por outros estudos que também controlaram as variáveis da área de contorno, densidade e área total ocupada contrastando-as com as informação de natureza numérica (Clearfield \& Mix, 2001; Feigenson et al., 2002). Além de lançar dúvidas sobre a hipótese inatista do conceito de número natural, estes estudos enfatizaram a necessidade crítica de se fazer controles das variáveis perceptivas e conceituais em estudos sobre as habilidades quantitativas dos bebês. Estudos recentes, ao exercitar tal controle de variáveis em seus experimentos, mostraram um cenário de processos cognitivos mais complexos, exigindo outras propostas explicativas da comunidade acadêmica que extrapolem a simples conjectura dual: da existência de um módulo de senso numérico inato de um lado, e da aquisição conceitual tardia de outro.

Por exemplo, seguindo a preocupação de controlar a co-variação de informações perceptivas e numéricas, $\mathrm{Xu}$ e colaboradores $(\mathrm{Xu}, 2003$; Xu \& Spelke, 2000; Xu, Spelke, \& Goddard, 2005) realizaram vários estudos os quais revelaram que os bebês de seis meses de idade atendem para informação numérica quando: (a) as informações de área total e densidade variam, mas os números permanecem constantes, e, (b) quando os números apresentados nos conjuntos estão em uma razão numérica na ordem de 1:2 -i.e., 4 vs. 8, 8 vs. 16, 16 vs. 32. Este comportamento, que condiz com a lei de Weber (a qual estipula a menor diferença que pode ser percebida entre dois estímulos), também foi observado em estudos com animais infra-humanos (Brannon, Abbott, \& Lutz, 2004; Cordes \& Brannon, 2008). Entretanto, nestes mesmos estudos de $\mathrm{Xu}$ e col., assim como em outras pesquisas (Lipton \& Spelke, 2003; Wood \& Spelke, 2005), bebês foram repetidamente incapazes de perceber mudanças numéricas em conjuntos com uma razão numérica menor do que 1:2, tais como 4 vs. 6, 8 vs. 12, 16 vs. 24.

Adicionalmente, outros pesquisadores obtiveram resultados que sugerem que os bebês demonstram ser capazes de discriminar quantidades que não condizem com a lei de Weber na razão 1:2 se os conjuntos apresentam quantidades menores do que quatro elementos (Feigenson \& Carey, 2003, 2005). Por exemplo, Feigenson e Carey (2003) usaram a metodologia da busca manual e demonstraram que os bebês de 12 a 14 meses foram capazes de discriminar entre 2 vs. 3, mas não 2 vs. 4. Neste estudo, os bebês, ao verem o experimentador colocar três brinquedos dentro de uma caixa e retirar somente dois, passaram mais tempo buscando algo dentro da caixa do que na situação de quatro brinquedos inseridos e dois retirados. Em posterior estudo, as mesmas autoras (Feigenson $\&$ Carey, 2005) usaram bolachas sendo inseridas uma-auma dentro de dois recipientes. Um recipiente ficou com 
uma quantidade maior e outro com uma quantidade menor, tal como, 1 vs. 2, 2 vs. 3, 1 vs. 3. Após assistirem a colocação da quantidade de bolachas nos dois recipientes, os bebês foram encorajados a engatinhar até os recipientes. Nesta situação, os bebês consistentemente se direcionaram para o recipiente contendo mais bolachas. Mas nas situações onde a quantidade de bolachas colocadas era maior do que quatro (ex. 2 vs. 4, 3 vs. 6, 1 vs. 4) não houve preferência pelo bebê em escolher o recipiente com maior quantidade.

Estes resultados combinados formam um cenário complexo sobre as habilidades quantitativas dos bebês, o qual sugere que há pelo menos três caracterizações deste processo: (a) os bebês atendem para pistas perceptivas, tais como área de contorno, densidade e área total, em contextos de conjuntos de figuras (bi-dimensional) com pequenas quantidades; entretanto, (b) os bebês também parecem atender para pistas numéricas, em contextos nos quais os estímulos perceptivos contrastam com as informações numéricas em conjuntos de objetos discretos com pequena quantidade de itens $(<)$; e, por último, (c) os bebês parecem perceber quantidade numérica quando comparam conjuntos de grandes quantidades nos quais suas diferenças são suficientemente estabelecidas em uma razão de 1:2 ou 1:4

Inicialmente, ao ler estas caracterizações, podemos imediatamente perceber contradições entre as hipóteses (a) e (b) e não entender como estas, então, relacionam-se com (c). Portanto, é necessário analisar como pesquisadores explicam estas caracterizações a partir de seus entendimentos da natureza dos processos cognitivos envolvidos.

Pesquisadores que assumem que desde o início as discriminações quantitativas dos bebês são baseadas em conhecimento numérico per se, pesquisadores da hipótese inatista, desconsideram a caracterização (a) e discutem as caracterizações (b) e (c). Estes pesquisadores argumentam que, possivelmente, pode haver dois sistemas usados pelos bebês na discriminação de quantidade. Isto é, um sistema para discriminação de pequenas quantidades, o qual parece ser preciso e tem um limite de até três elementos denominado sistema de individuação de objetos (Feigenson \& Carey, 2003, 2005; Uller et al., 1999). E outro sistema para discriminação de grandes quantidades, o qual é aproximado e necessita de que as diferenças sejam grandes o suficiente para serem percebidas, seguindo uma razão proporcional de 1:2 (Cordes \& Brannon, 2008; Xu \& Spelke, 2000). Assim, quando devidamente controladas, as variáveis para que haja um contraste entre a informação perceptiva e a informação numérica, os bebês atendem para as informações numéricas preferencialmente e não para as informações perceptivas na discriminação de pequenas quantidades. Quando o contexto experimental exige a discriminação de grandes quantidades, então os bebês se utilizam do mecanismo de representação aproximada de magnitudes.
Portanto, os mecanismos apontados por este grupo de pesquisadores pelos quais os bebês são capazes de discriminar quantidade numéricamente são: (a) individuação de objetos para pequenas quantidades e (b) representação de magnitude para grandes quantidades. O mecanismo de representação de magnitude opera de forma aproximada no julgamento de quantidades global dos conjuntos e necessita de razões numéricas suficientemente altas para estabelecer as diferenças (lei de Weber). Estudos têm apontado que este mecanismo é também operacional em animais vertebrados (Brannon \& Terrace, 2000), sugerindo, assim, uma origem filogenética para um mecanismo do processo ontogenético da cognição matemática. Há sugestões de que esta representação de magnitudes possa funcionar como um acumulador de impulsos (Gelman, 1991; Wynn, 1998b). Desta forma, tanto as pessoas quanto os animais criam representações aproximadas para cada conjunto, aumentando a representação em correspondência à magnitude apresentada; por exemplo, para um conjunto com a quantidade $/ 6 /$ o acúmulo representado pode ser assim /__ / e para um conjunto com a quantidade $/ 10 /$ pode ser assim /

Já o mecanismo de individuação opera para os objetos discretos que fazem parte do conjunto (e não conjuntos) e atribui uma representação mental (como se fosse um símbolo) para cada objeto. Entretanto, devido ao limite da memória de trabalho do bebê, este mecanismo tem o limite de operar com até três objetos. A hipótese original de Uller et al. (1999) especulava que por esse mecanismo ocorreria uma representação da localização espacial do objeto, mas nas formulações recentes (Le Corre \& Carey, 2007) o mecanismo é o da representação de objetos individuais na memória de trabalho.

Em uma recente análise da literatura (Rips, Bloomfield, \& Asmuth, 2008) sobre as implicações destes dois sistemas de discriminação quantitativa (para pequenas e grandes quantidades) e dos diferentes mecanismos que viabilizam a formação de representações mentais numéricas (individuação de objetos e representação de magnitudes), pesquisadores argumentaram que estes estudos ainda não demonstraram como essas representações numéricas iniciais (inatas) podem levar à construção do conceito de número natural. Se as crianças partem da trilha dos mecanismos descritos acima, não há razão para que seja concluído que elas têm um conceito inato de número natural. Uma vez que, nem o mecanismo de representação de magnitude e nem mesmo o mecanismo de representação mental de objetos individualizados têm as propriedades dos números naturais. As propriedades que definem os números naturais (para uma revisão ver Wiese, 2003) são: as entidades são distintas, elas formam uma progressão e esta progressão é infinita. Desta forma, os números naturais são definidos pelas relações que constituem a progressão infinita e não por representações instanciadas de quantidades. 
Assim, mesmo que os autores destes estudos aqui revisados afirmem que seus dados mostram a existência de "conceitos numéricos" ou "conceitos de número", podemos afirmar que o quê os estudos até o momento nos revelaram foi a presença de ideias quantitativas pelos quais os bebês e as crianças pequenas fazem julgamentos com base em objetos, mas, não a existência nos bebês de um conceito de número natural através do qual eles conseguiriam estabelecer que $1<2$ e que para cada número há sempre um maior dentro de uma progressão ordenada e infinita (propriedades dos números naturais). É importante que a diferença entre "conceitos numéricos" e "conceito de número natural" fique clara dentro do escopo destas pesquisas.

Mas, considerando - como exercício analítico - que as crianças construam um conceito de número natural a partir dos mecanismos cognitivos propostos acima, será necessário então um processo de mudança das estruturas cognitivas existentes para que seja possível uma verdadeira abstração conceitual de número; pois, como foi sugerido acima, as representações quantitativas feitas através destes mecanismos cognitivos não trazem em si as propriedades dos números naturais. $\mathrm{O}$ presente desafio dos pesquisadores é, então, esclarecer os processos e habilidades cognitivas envolvidas nesta mudança.

O quê também parece ficar de fora tanto das explicações destes pesquisadores, quanto da crítica recentemente elaborada, é o fato de que as metodologias usadas para a coleta de dados nestes estudos experimentais em nada se relacionam com o contexto real em que as crianças aprendem sobre números. Nos estudos experimentais os objetos aparecem e desaparecem de forma mágica, ou ainda, são apresentados de forma isolada. No contexto de vida real, os objetos são apresentados, tocados, contados, agrupados, separados dentro de contextos de interação e intencionalidade. Assim sendo, as habilidades evidenciadas dentro do contexto experimental podem refletir a preferência de uso por um ou outro mecanismo devido ao próprio design do experimento, o qual não permite o uso de processos paralelos. Portanto, sem querer diminuir a importância dos estudos experimentais, talvez o que temos de seus resultados é uma parte (valiosa parte) de um todo mais complexo.

Um segundo problema com as explicações oferecidas ainda por este grupo de pesquisadores é a ideia de que os conceitos de número são fenômenos monolíticos, os quais só podem ser explicados através de um único fator: informação numérica. E esta visão monolítica ocorre tanto pela via de que dentre todos os fatores envolvidos no conceito de número somente um, a informação numéri-

\footnotetext{
${ }^{5}$ Acredita-se que o termo mais apropriado seria "ideias quantitativas" para descrever os resultados dos estudos cognitivos nesta área, mas os pesquisadores desses estudos usam o termo "conceitos" em suas conclusões. Assim, para ser fiel à terminologia empregada pelos pesquisadores cognitivistas usa-se o termo conceito no plural, mas faz-se esta ressalva.
}

ca, é o que realmente importa; quanto, alternativamente, pela via de que todos os diversos fatores envolvidos no conceito de número são, na verdade, diferentes instâncias de um mesmo fator: informação numérica. Esta visão monolítica de uma cognição descontextualizada ameaça um entendimento mais compreensivo dos processos cognitivos envolvidos na construção de conceito de número natural.

A partir desta crítica, podemos perguntar: o bebê usa somente uma trilha para representar quantidade? Ou, ele usa também a trilha da percepção de área ocupada, da representação de magnitude e da individuação de objetos? Não poderia a criança fazer uso de diferentes informações (trilhas) dependendo do contexto, dos materiais usados, da demanda da situação e do seu desenvolvimento?

A resposta simples é sim. Alguns pesquisadores têm inclinado suas interpretações dos resultados de pesquisas sobre cognição numérica nesta direção.

\section{Vários Precursores Envolvidos na Trilha de Objetos a Número}

De acordo com um grupo de pesquisadores que discute o desenvolvimento cognitivo a partir de uma visão pela qual os processos gerais cognitivos tornam-se especializados no decorrer do desenvolvimento, o conceito de número natural pode ter como precursores processos gerais básicos. Estes processos cognitivos de domínio geral auxiliam o desenvolvimento de várias habilidades cognitivas que participam na formação do conceito de número natural (Clearfield \& Mix, 1999, 2001; Huttenlocher et al., 2002; Mix, Sandhofer, \& Baroody, 2005; Wakeley, Rivera, \& Langer, 2000). De acordo com esta perspectiva, alguns processos de domínio geral que participam da conceituação numérica são: a habilidade perceptiva do bebê que auxilia nos processos de individuação do objeto e de percepção de magnitudes, a habilidade sócio-interativa, a habilidade lingüística (aquisição dos nomes dos numerais), os processos básicos de memória, atenção e associação. Esses processos cognitivos gerais podem causar a focalização da atenção nos aspectos numéricos e, conseqüentemente, podem levar à formação do conceito de número sem ser necessário evocar a existência de um módulo numérico inato e específico para o processamento somente de informações de base numérica.

Estes pesquisadores afirmam que a metodologia de habituação não demonstra, indubitavelmente, que o comportamento do bebê de olhar mais intensamente para algo reflete um conhecimento pré-existente deste algo, pois o próprio ato de habituar pode tornar relevantes os aspectos ou as variáveis que antes não eram relevantes (Mix et al., 2002a). Ou seja, expor o bebê a um estímulo que se repete (chegando a 30 vezes) pode criar uma atenção ao aspecto numérico que não existia antes. Portanto, isso não quer dizer que o bebê tenha a capacidade inata de perce- 
ber número, mas talvez o aspecto quantitativo-numérico passe a ser relevante dentro do próprio contexto do experimento que isola e enfatiza este aspecto. Os estudos de Clearfield e Mix (1999, 2001), como já discutidos anteriormente, confirmaram que os bebês são sensíveis às mudanças de densidade, comprimento e ocupação espacial (perceptivas), e não às mudanças numéricas. Reproduções do estudo de Clearfield e Mix obtiveram os mesmos resultados (Feigenson \& Carey, 2003, 2005; Feigenson et al., 2002).

Assim, a explicação inatista original de que conhecimento numérico inato guia o desempenho dos bebês em atividades de discriminação de quantidades perde sua força com as recentes evidências que demonstraram que os bebês, na verdade, também usam informações perceptivas e espaciais para elaborar representações de individuação e agrupamento dos mesmos (Cantlon, Platt, \& Brannon, 2009; Cordes \& Brannon, 2008; Mix \& Sandhofer, 2007). Esta explicação tem sido abandonada, de modo geral, pela comunidade acadêmica.

Mas uma nova conjectura tomou o lugar dessa proposta original: é a ideia de que as representações mentais de magnitude e de objetos discretos, assim como outros fatores, são capacidades que se desenvolvem ao longo do tempo e as mesmas desempenham importantes papeis nos processos que guiam a formação do conceito de número (Cantlon et al., 2009; Carey, 2008; Mix, 2008; Sophian, 2008). Desta forma, as representações mentais criadas pelos bebês humanos que envolvem quantidade não são, necessariamente, guiadas por um conceito inato de número, mas podem ser representações quantitativo-numéricas advindas das habilidades perceptivas gerais e que podem posteriormente influenciar o desenvolvimento do conceito de número natural.

\section{Propostas de Como os Bebês Constroem Conceito de Número a Partir das Habilidades Iniciais?}

Das representações quantitativas iniciais para a contagem verbal ocorre um longo e complexo processo de desenvolvimento. A aquisição dos nomes dos numerais, como também, a aquisição dos procedimentos de contagem e o entendimento de porquê e o quê contar, requer a junção de vários conhecimentos de ordem conceitual e prática de parte do sujeito cognoscente.

Wynn (1992a, 1992b) usou duas tarefas simples para coletar dados sobre como se caracteriza o conceito de número das crianças de dois anos e meio à cinco anos de idade. A primeira tarefa foi "Me dar N": apresentava-se às crianças uma cesta de dinossauros e, então, o pesquisador lhes solicitava que lhe dessem um determinado número de bichos ("Me dê dois dinossauros"). A segunda tarefa consistia em a criança identificar, entre duas cartas contendo fotos de conjuntos de objetos, qual delas correspondia a uma determinada quantidade; por exemplo, "Você pode me mostrar três maçãs?". Ambas as tarefas não apresentavam grande grau de dificuldade, eliminando-se, assim, a possibilidade de problemas no desempenho durante os testes. Wynn também pediu que as crianças contassem um conjunto com oito objetos. Os resultados dos dois estudos de Wynn (um de corte transversal e o outro longitudinal) mostraram que, apesar de as crianças terem sido capazes de contar corretamente pelo menos até seis, elas não sabiam o valor cardinal exato de todos os números na sua lista de contagem oral. Por exemplo, quando solicitadas para produzir e identificar uma quantidade, algumas crianças (geralmente as mais novas) só conseguiam produzir e identificar a quantidade "um" com exatidão; outras (um pouco mais velhas) somente "um e dois", outras somente "um, dois e três". Wynn também mostrou que demora mais ou menos seis meses o período entre a aquisição do significado cardinal de um numeral para outro, isto é, a criança primeiramente constrói o conceito de "um", depois de "dois" e depois de "três" de forma gradual. Adicionalmente, a autora mostrou que a maioria das crianças não usa espontaneamente a contagem oral para solucionar problemas e, também, comete erros de contagem que violam princípios de correspondência um-para-um.

Os estudos de Wynn (1992a, 1992b), portanto, sugerem que inicialmente a contagem oral é, como havia sugerido anteriormente Piaget e Szeminska (Piaget, 1952) e Fuson (1988), um procedimento aprendido no contexto sociocultural da criança. Mas Wynn, todavia, argumenta que para o conceito de número se desenvolver é necessário que o mesmo seja precedido e sustentado por mecanismos cognitivos de representações numéricas mentais, os quais, a autora afirma, são de natureza inata. A hipótese de Wynn é a de que as palavras "um" "dois" "três" "quatro", por exemplo, são rótulos que, ao ressaltarem o aspecto numérico, precisam ser casados com as representações mentais numéricas anteriormente construídas pelo sentido de número ${ }^{6}$ inato que todo o bebê humano possui.

Mas, há outras hipóteses sobre como as crianças podem construir o conceito de número natural a partir das habilidades iniciais dos bebês. Segundo os neurocientistas Cohen Kadosh e Walsh (2008), há uma grande participação da área do sulco intraparietal (IPS) em atividades desenvolvidas na representação mental numérica, como também, em atividades de representação de outras magnitudes tais como ocupação de espaço físico, duração temporal, densidade e luminosidade. Os resultados de vários estudos desenvolvidos por estes neurocientistas possibilitaram aos mesmos argumentar que o lobo parietal é a base para um sistema comum de representação de magnitudes tanto para número, quanto para tempo e espaço. Portanto, não há exclusividade do sistema para números.

6 O termo "sentido de número" foi introduzido por $\mathrm{S}$ Dehaene (1997), o qual defende que os bebês possuem um sentido de número inato. 
Pesquisas no nível de comportamento realizadas no laboratório de Elizabeth Brannon (ver, Cantlon et al., 2009) também mostram que o mesmo sistema de representação de magnitude é usado para representar número, tempo e espaço.

Assim, podemos argumentar que há processos gerais de representação de magnitudes, sob os quais são criadas representações mais específicas. Cohen Kadosh e Walsh (2008) sugerem que o conceito de número natural surge da interação de substratos neuronais envolvidos em representações gerais de magnitudes com as áreas do hemisfério esquerdo envolvidas na linguagem e com as áreas do sistema ventral-ocipto-temporal envolvidas em processamento simbólico. Esta visão interativa que compreende várias regiões cerebrais e processos cognitivos na construção de funções especializadas é denominada de abordagem de especialização interativa. Segundo esta abordagem, o conceito de número emerge da interação de processos gerais de representação de magnitude, linguagem e habilidade simbólica. Uma proposta muito semelhante à de Cohen Kadosh e Walsh é apresentada por Mix e Sandhofer (2007).

Com essas novas conjecturas, a lição que estamos começando a aprender é de que talvez não haja uma só via para se chegar do ponto $A$ ao ponto $B$. Pode ser que o processo de desenvolvimento do conceito de número seja múltiplo e variado. Pesquisadores têm argumentado que apesar desta variação ser intrínseca ao processo de desenvolvimento humano, a mesma é mais visível na criança devido à natureza de sua aprendizagem, a qual é marcada pelo contexto (Mix, 2002; Nelson, 1996; Thelen \& Smith, 1994). Assim, é possível que a criança exiba um comportamento mais competente dentro de um contexto, e menos competente dentro de outro contexto, dependendo dos instrumentos de suporte a que a criança tem acesso. Estes instrumentos de suporte são: a linguagem, objetos do mundo físico, organização do espaço físico, interações sociais. O desenvolvimento cognitivo, então, passa a ser entendido como os processos de criação de ligações entre estes contextos de aprendizagem que podem inicialmente estar separados (Barbosa, 2004; Baroody, 2003). Desta forma, podemos pensar o desenvolvimento de conceito de número natural como um processo de criação de conexões e relações flexíveis entre habilidades de caráter quantitativo-numéricas e demais habilidades cognitivas.

\section{Considerações Finais}

Em conclusão, podemos sugerir que a hipótese piagetiana tem sido corroborada em estudos recentes que mostraram a fragilidade dos argumentos inatistas e a necessidade de investigação mais cuidadosa sobre a complexidade envolvida na construção do conceito de número natural pela criança. Assim, as evidências analisadas sugerem que o conceito de número natural não é inato. Mas, fruto de processo de desenvolvimento complexo e gradual que envolve de forma dinâmica os fatores biológicos e culturais.

Futuramente, precisamos investigar de forma mais profunda o relacionamento entre a habilidade lingüística e a construção do conceito de número natural. Pois, sendo a linguagem uma precursora deste conceito, é imperativo entendermos que mudanças cognitivas são efetivadas a partir da aquisição da linguagem na construção do conceito de número.

Precisamos também pensar em novas metodologias no estudo da cognição matemática. Nós vimos que a metodologia de habituação é a mais comumente usada com bebês; já a metodologia de tarefas experimentais é a mais comumente usada com crianças entre dois e seis anos de idade. Ambas as metodologia tem méritos e também problemas. Talvez devêssemos buscar combinar as metodologias experimentais com metodologias mais naturalistas. Por exemplo, já existem estudos que utilizam a metodologia microgenética para descrever processos detalhados de desenvolvimento. Para a metodologia microgenética, as habilidades da criança devem ser entendidas dentro do contexto particular de sua realização (Siegler, 1995, 1996). Esta proposta metodológica tem se apresentado como uma importante metodologia para estudar os processos de desenvolvimento e aprendizagem.

É importante ainda perceber que a criança real, presente nas casas e nos centros de educação infantil, não é a criança dos laboratórios de pesquisa, que em idade $x$ faz $w$ e em idade $y$ faz $m$. As crianças exibem uma multiplicidade de comportamentos, estratégias e capacidades que podem ser expressas simultânea e separadamente e que, também, podem ser mais sofisticadas ou menos sofisticadas de acordo com o contexto. A construção do conceito de número natural a partir de habilidades quantitativas iniciais é um processo gradual, variável, e, possivelmente, atrelado ao contexto onde esta ocorre.

\section{Referências}

Antell, S., \& Keating, D. P. (1983). Perception of numerical invariance in neonates. Child Development, 54, 695-701.

Barbosa, H. (2004). Numerical abilities of preschool children with atypical development: A developmental description. (Unpublished doctoral dissertation). Boston University, MA. Baroody, A. J. (2003). The development of adaptive expertise and flexibility: The integration of conceptual and procedural knowledge. In A. J. Baroody \& A. Dowker (Eds.), The development of arithmetic concepts and skills: Constructing adaptive expertise (pp. 1-33). Mahwah, NJ: Lawrence Erlbaum.

Brannon, E. M., Abbott, S., \& Lutz, D. J. (2004). Number bias for the discrimination of large visual sets in infancy. Cognition, 86(3), B59-B68.

Brannon, E. M., \& Terrace, H. S. (2000). Representation of numerosities 1-9 by rhesus macaques (Macaca mulatta). Journal of Experimental Psychology: Animal Behavior Processes, 26(1), 31-49. 
Barbosa, H. H. (2012). Das Competências Quantitativas Iniciais para o Conceito de Número Natural: Quais as Trilhas Possíveis?

Butterworth, B. (1999). What counts: How every brain is hardwired for math. New York: The Free Press.

Cantlon, J. F., Platt, M. L., \& Brannon, E. M. (2009). Beyond the number domain. Trends in Cognitive Science, 13(2), 83-91.

Carey, S. (1991). Knowledge acquisition: Enrichment or conceptual change? In S. Carey \& R. Gelman (Eds.), The epigenesis of mind: Essays on biology and cognition (pp. 257-291). Hillsdale, NJ: Lawrence Erlbaum.

Carey, S. (2008). Math schemata and the origins of number representations. Behavioral and Brain Sciences, 31(6), 645 646.

Carey, S., \& Spelke, E. (1994). Domain-specific knowledge and conceptual change. In L. A. Hirschfield \& S. Gelman (Eds.), Mapping the mind: Domain specificity in cognition and culture (pp. 169-200). New York: Cambridge University Press.

Clearfield, M. W., \& Mix, K. S. (1999). Number versus contour length in infants' discrimination of small visual sets. Psychological Science, 10(5), 408-411.

Clearfield, M. W., \& Mix, K. S. (2001). Amount versus number: Infants' use of area and contour length to discriminate small sets. Journal of Cognition and Development, 2, 243-260.

Cohen Kadosh, R., \& Walsh, V. (2008). From magnitude to natural numbers: A developmental neurocognitive perspective. Behavioral and Brain Sciences, 31(6), 647-648.

Cordes, S., \& Brannon, E. (2008). Quantitative competencies in infancy. Developmental Science, 11(6), 803-808.

Dehaene, S. (1997). The number sense: How the mind creates mathematics. New York: Oxford University Press.

Feigenson, L., \& Carey, S. (2003). Tracking individuals via object-files: Evidence from infants' manual search. Developmental Science, 6, 568-584.

Feigenson, L., \& Carey, S. (2005). On the limits of infants' qualification of small object arrays. Cognition, 97(3), 295313.

Feigenson, L., Carey, S., \& Spelke, E. (2002). Infants' discrimination of number vs. continuous extent. Cognitive Psychology, 44(1), 33-66.

Fuson, K. (1988). Children's counting and concepts of number. New York: Springer-Verlag.

Gelman, R. (1991). Epigenetic foundations of knowledge structures: Initial and transcendent constructions. In S. Carey \& R. Gelman (Eds.), The epigenesis of mind: Essays on biology and cognition (pp. 293-322). Hillsdale, NJ: Lawrence Erlbaum

Gelman, R., \& Gallistel, C. R. (1978). The child's understanding of number. Cambridge, MA: Harvard University Press.

Gelman, R., \& Meck, E. (1983). Preschoolers' counting: Principles before skill. Cognition, 13, 343-359.

Huttenlocher, J., Duffy, S., \& Levine, S. (2002). Infants and toddlers discriminate amount: Are they measuring? Psychological Science, 13, 244-249.

Le Corre, M., \& Carey, S. (2007). One, two, three, four, nothing more: An investigation of the conceptual sources of the verbal counting principles. Cognition, 105, 395-438.

Lipton, J. S., \& Spelke, E. (2003). Origins of number sense: Large-number discrimination in human infants. Psychological Science, 14, 396-401.

Mix, K. S. (2002). The construction of number concepts. Cognitive Development, 17, 1345-1363.

Mix, K. S. (2008). The origins of number: Getting developmental. Behavioral and Brain Sciences, 31(6), 662.
Mix, K. S., Huttenlocher, J., \& Levine, S. C. (2002a). Quantitative development in infancy and early childhood. New York: Oxford University Press.

Mix, K. S., Huttenlocher, J., \& Levine, S. C. (2002b). Multiple cues for quantification in infancy: Is number one of them? Psychological Bulletin, 128, 278-294.

Mix, K., \& Sandhofer, C. (2007). Do we need a number sense? In M. J. Roberts (Ed.), Integrating the mind (pp. 293-326). Hove, UK: Psychology Press.

Mix, K., Sandhofer, C., \& Baroody, A. (2005). Number words and number concepts: The interplay of verbal e non-verbal quantification in early chilhood. In R. V. Kail (Ed.), Advances in Child Development and Behavior (Vol. 33, pp. 305-346). New York: Academic Press.

Nelson, K. (1996). Language in cognitive development: Emergence of the mediated mind. New York: Cambridge University Press.

Piaget, J. (1952). The child's concept of number. New York: Norton.

Rips, L. J., Bloomfield, A., \& Asmuth, J. (2008). From numerical concepts to concepts of number. Behavioral and Brain Sciences, 31(6), 623-642.

Siegler, R. S. (1995). How does change occur: A microgenetic study of number conservation. Cognitive Psychology, 28, 225273.

Siegler, R. S. (1996). Emerging minds: The process of change in children's thinking. New York: Oxford University Press.

Sophian, C. (2008). Precursors to number: Equivalence relations, less-than and greater-than relations and units. Behavioral and Brain Sciences, 31(6), 670-671.

Spelke, E., \& Dehaene, S. (1999). Biological foundations of numerical thinking: Response to T. J. Simon. Trends in Cognitive Sciences, 10, 365-366.

Spelke, E., \& Tsivkin, S. (2001). Initial knowledge and conceptual change: Space and number. In M. Bowerman \& C. Levinson (Eds.), Language acquisition and conceptual development (pp. 70-97). Cambridge, UK: Cambridge University Press.

Starkey, P., \& Cooper, R. G., Jr. (1980). Perception of number by human infant. Science, 210, 1033-1035.

Starkey, P., \& Spelke, E. (1983). Detection of intermodal numerical correspondences by human infants. Science, 222 , 179-181.

Starkey, P., Spelke, E., \& Gelman, R. (1990). Numerical abstraction by human infants. Cognition 36, 97-127.

Strauss, M. S., \& Curtis, L. E. (1981). Infants perception of numerosities. Child Development, 52, 1146-1152.

Strauss, M. S., \& Curtis, L. E. (1984). Development of numerical concepts in infancy. In C. Sophian (Ed.), Origins of cognitive skills: The Eighteenth Annual Symposium on Cognition (pp. 131-155). Hillsdale, NJ: Lawrence Erlbaum.

Thelen, E., \& Smith, L. B. (1994). A dynamic systems approach to the development of cognition and action. Cambridge, MA: The MIT Press.

Uller, C., Carey, S., Huntley-Fenner, G., \& Klatt, L. (1999). What representations might underlie infant numerical knowledge? Cognitive Development, 14, 1-36.

Van de Walle, G., Carey, S., \& Prevor, M. (2000). Bases for object individuation in infancy: Evidence from manual search. Journal of Cognition and Development, 1, 249-280.

Wakeley, A., Rivera, S., \& Langer, J. (2000). Can young infants add and subtract? Child Development 71, 1525-1534.

Wiese, H. (2003). Numbers, language and the human mind. Cambridge, UK: Cambridge University Press. 
Wood, J. N., \& Spelke, E. (2005). Infants' enumeration of action: Numerical discrimination and its signature limits. Developmental Science, 8, 173-181.

Wynn, K. (1992a). Children's acquisition of the number words and the counting system. Cognitive Psychology, 24, 220-251.

Wynn, K. (1992b). Addition and subtraction by human infants. Nature, 358, 749-750.

Wynn, K. (1992c). Evidence against empiricist accounts of the origins of numerical knowledge. Mind and Language, 7, 315332.

Wynn, K. (1998a). Psychological foundations of number. Trends in Cognitive Science, 2, 296-303.

Wynn, K. (1998b). Numerical competence in infants. In C. Donlan (Ed.), The development of mathematical skills. Studies in Developmental Psychology (pp. 3-25). Hove, England: Psychology Press.

$\mathrm{Xu}, \mathrm{F}$. (2003). Numerosity discrimination in infants: Evidence for two systems of representations. Cognition, 89, B15-B25.

$\mathrm{Xu}, \mathrm{F}$., \& Spelke, E. (2000). Large number discrimination in 6month old infants. Cognition, 74, B1-B11.

$\mathrm{Xu}$, F., Spelke, E., \& Goddard, S. (2005). Number sense in human infants. Developmental Science, 8(1), 88-101. 